\title{
LA EDUCACIÓN FÍSICA Y EL DEPORTE COMO HERRAMIENTAS DE INCLUSIÓN DE LA POBLACIÓN INMIGRANTE EN CATALUÑA: EL PAPEL DE LA ESCUELA Y LA ADMINISTRACIÓN LOCAL
}

\author{
Susanna Soler Prat \\ Instituto Nacional de Educación Física de Cataluña, Barcelona, España
}

Gonzalo Flores Aguilar

Universidad Autónoma de Barcelona, Barcelona, España

Maria Prat Grau

Universidad Autónoma de Barcelona, Barcelona, España

\begin{abstract}
Resumen:
Hay dos instituciones que juegan un papel clave para fomentar la inclusión de la población inmigrante por su proximidad con la ciudadanía: la escuela y la administración local. En este artículo, se exponen las principales actuaciones que se llevan a cabo desde ambos terrenos para aprovechar el potencial socializador del deporte y la actividad física en Cataluña. Partiendo del planteamiento político que guían las actuaciones educativas y en ámbito municipal, se describen algunas experiencias en las dos esferas. Fruto de este análisis, se constata la importancia de diseñar las estrategias y propuestas de intervención teniendo en cuenta los principios básicos de participación, transversalidad y continuidad.
\end{abstract}

Palabras clave: Educación Física. Deporte. Inmigración. Inclusión.

\section{Introducción}

Cataluña ha sido, desde tiempos antiguos y hasta la actualidad, un país de "encuentro" de pueblos y culturas. Su situación geográfica, a orillas del Mediterráneo y entre España y Europa, junto con su desarrollo económico y social, la han convertido en una tierra propicia a la llegada de nuevos habitantes y a la amalgama cultural.

Durante la segunda mitad del siglo XX y en los inicios del siglo XXI, Cataluña ha vivido un gran cambio de población fruto de dos grandes oleadas migratorias: en primer lugar, en los años 60, llegó a Cataluña un gran número de población procedente de otras partes de España; más adelante, a finales de los años 90, llegó una segunda oleada de población procedente, en esta ocasión, de otros países.

Este movimiento migratorio ha llevado a un importante y rápido incremento de la población en Cataluña que, según los censos munici- 
pales, ha pasado de los 6.261.999 habitantes en el 2000, con un $3 \%$ de población extranjera aproximadamente, a 7.512.381 en el 2010, con un $16 \%$ de habitantes procedentes de otros países, especialmente, de países en vías de desarrollo de Iberoamérica y del norte de África (IDESCAT, 2011).

Ante esta mayor heterogeneidad sociocultural, tal y como recoge el Libro Blanco del Deporte, elaborado por la Comisión Europea en 2007, la actividad física y el deporte se convierten en espacios privilegiados para favorecer la inclusión social de la población inmigrante así como la convivencia y el respeto a la diversidad. En este marco, como expone Gaspar Maza (2002), el deporte puede convertirse en un instrumento para evitar el esquema de reproducción social de la inmigración extrajera situada en riesgo de exclusión así como de la población general situada en la marginación. Sin embargo, como señalan constantemente la mayor parte de especialistas, el deporte y la actividad física tienen también sus limitaciones y sería un grave error considerarlos la panacea de las problemáticas existentes en las sociedades actuales.

En esta ambivalencia, hay dos instituciones que juegan un papel clave: la escuela y la administración local, las más cercanas a la ciudadanía. En este artículo, se exponen las principales actuaciones que se llevan a cabo desde ambas instituciones para aprovechar el potencial socializador del deporte y la actividad física en Cataluña.

\section{La escuela: la atención al alumnado inmigrante y la educación in- tercultural desde la Educación Física}

La consecuencia más inmediata de la intensificación de los flujos migratorios que se han dado en Cataluña en estos últimos años ha sido la diversificación étnica y cultural de la población escolar. En el curso escolar 2010-2011, según datos del Departament d'Educació (2011), el 13,7\% del alumnado de educación primaria matriculado en la escuela catalana procedía de otro país, además del alumnado de padres inmigrantes nacido en Cataluña (no recogido estadísticamente).

Este importante incremento, especialmente en los últimos años, y la llegada gradual de nuevo alumnado a lo largo del curso, ha generado un cambio importante en el día a día de los centros educativos y una mayor complejidad. Así, la escuela ha tenido que afrontar un auténtico reto para conseguir ser realmente un agente inclusivo y sociali- 
zador para un alumnado de procedencias muy variadas y un espacio desde el que se fomente el respeto y la convivencia.

Para ello, en el marco de la Ley de Educación de Cataluña (LEC), elaborada en el 2009 con la intención de mejorar la calidad del sistema educativo catalán y dotarlo de estabilidad, se revisó y actualizó el "Plan para la Lengua y la Cohesión social" (PLCS), elaborado en el 2004. El PLCS ofrece diferentes líneas de trabajo a los centros para que fomenten la cohesión social en la escuela a través de la consolidación de la lengua catalana, el fomento de la educación intercultural, y la promoción de la igualdad de oportunidades (DEPARTAMENT D’EDUCACIÓ, 2009b).

Para la consecución de dichos objetivos, la implementación de este plan en la escuela originó, entre otras novedades, la creación del Aula de acogida. Es en esta Aula de acogida donde, de forma segregada, se atiende al alumnado que no domina el catalán para potenciar sus competencias lingüísticas hasta que tiene un nivel suficiente de comprensión y comunicación en catalán o castellano ${ }^{1}$.

Sin embargo, para facilitar su progresiva socialización, durante las clases de las asignaturas más instrumentales - la Educación Física, la Música y la Educación Plástica -, el alumnado se integra al grupo ordinario correspondiente. Así, la Educación Física se convierte aún más en una herramienta de primer orden para facilitar la inclusión del alumnado inmigrante y el desarrollo de la educación intercultural. A continuación se describe su papel.

\section{¿Hacia una Educación Física intercultural?}

¿A qué nos referimos cuando hablamos de educación intercultural? Como argumentan García y Sales (1997), la educación intercultural parte del reconocimiento y respeto hacia la diversidad para propiciar el enriquecimiento cultural de los ciudadanos y ciudadanas a través del intercambio, el diálogo y la participación activa y crítica, lo cual puede favorecer el desarrollo de una sociedad democrática basada en la igualdad, la tolerancia y la solidaridad.

1-El catalán es la lengua oficial de Cataluña -junto con el castellano- y la lengua vehicular en la escuela. Un grupo de especialistas de "Lengua, Interculturalidad y Cohesión social" (LIC), dotan al profesorado tutor del aula de acogida de una serie de recursos y medios, también disponibles en espacio web (http://www.xtec.net/lic/index.htm). 
En esta línea, las directrices curriculares del área de Educación Física en educación primaria no dudan en resaltar, como punto de partida, que las actividades que se promuevan en esta asignatura "tienen que potenciar las actitudes y valores propios de una sociedad solidaria, sin discriminación, respetuosa con las personas y el entorno" (DEPARTAMENT D’EDUCACIÓ, 2009a, p. 116).

Teniendo en cuenta este principio, la Educación Física contribuye especialmente al desarrollo de la educación intercultural mediante dos competencias recogidas en su currículum: la competencia comunicativa, centrada en la experimentación del cuerpo y el movimiento como instrumento de expresión y comunicación; y la competencia social y ciudadana, en la que se trabaja la adquisición de valores como el respeto, la aceptación y la cooperación a través de las relaciones interpersonales, el aprendizaje de reglas para el funcionamiento colectivo, el respeto hacia las diferencias y, en definitiva, el aprender a convivir.

Por otra parte, en el currículum también aparece un objetivo claramente orientado al conocimiento y al respeto de la diversidad cultural: "Conocer y valorar la diversidad de actividades físicas, lúdicas y deportivas como elementos culturales, propios y de otras culturas, mostrando una actitud crítica tanto de la perspectiva de participante, como de espectador/a" (DEPARTAMENT D'EDUCACIÓ, 2009a, p. 119).

Sin embargo, para que los propósitos establecidos en el currículum se lleguen a materializar, es necesario que el profesorado de Educación Física lleve a cabo una intervención intencionada para aprovechar el potencial inclusor de su asignatura. El deporte y la actividad física no educan en valores ni integran por sí solos sino que son las personas que los utilizan como herramienta quines tienen este poder. Como señala Heinemann (2002), son los individuos o las instituciones las que le asignan un determinado valor al deporte (económico, educativo, social, etc.) pero este, en sí mismo, no tiene unos valores intrínsecos. En esta línea, la investigación de Capllonch, Godall y Lleixà (2007) muestra como el maestro o la maestra debe incidir expresamente en este sentido si realmente quiere contribuir al enriquecimiento cultural y a la cohesión social en nuestras aulas. A continuación se señalan algunas de las consideraciones a tener especialmente en cuenta en la atención al alumnado inmigrante y recién llegado.

Sin duda, el hecho de que el desconocimiento del idioma no sea una barrera tan relevante como en otras áreas, facilita la tarea del profesorado. No obstante, debemos tener muy en cuenta que el lenguaje 
corporal también tiene códigos culturales muy potentes que pueden llevar a la confusión, generar malentendidos, o incluso suscitar situaciones de intimidación, en el caso, por ejemplo, de invadir el espacio personal de la otra persona.

Por otra parte, su carácter vivencial y lúdico hace que una buena parte del alumnado muestre un alto grado de motivación y sea un espacio idóneo para la educación en valores. A pesar de ello, como se ha señalado a menudo (PRAT; SOLER, 2002, 2003; SOLER, 2009), aquellos niños y niñas que no se sienten hábiles motrizmente pueden experimentar situaciones comprometidas y de fracaso que no le ayuden a su inclusión en el grupo ni a reforzar su autoestima. Es importante, entonces, generar contextos de aprendizaje seguros en que se pueda aprender sin temor a los reproches o burlas del resto del grupo, $\mathrm{y}$ en el que se resuelvan las situaciones de conflicto que puedan surgir en el transcurso de la sesión.

Otro de los argumentos habituales para considerar la Educación Física como una herramienta privilegiada es el hecho de que muchas de las actividades que se llevan a cabo (juegos, deportes, ejercicios, danzas, etc.) son universales y se realizan en todas partes. Pero para que ello suceda y se pueda disfrutar de un amplio espectro de actividades, es necesaria la diversificación de los contenidos y evitar el predominio de los más hegemónicos.

Precisamente para conseguir una mayor diversificación de las actividades, y con la voluntad de educar hacia la interculturalidad, en los últimos años se observa la tendencia a introducir juegos y danzas del mundo en las clases de Educación Física. Para ello, ha surgido un amplio repertorio de publicaciones en las que se pueden encontrar fichas y recursos para llevarlos a cabo (AGUDO et al., 2002; BANTULÀ; MORA, 2002; GARCÍA; MARTÍNEZ, 2004; GRANDA et al., 1999; MORA; DÍEZ; LLAMAS, 2003; SÁNCHEZ; MARTÍN, 2002; VELÁZQUEZ, 2006).

Como muestran diversas experiencias educativas (CUEVAS, 2009; EXPÓSITO et al. 1999; ORTÍ, 2005; VELÁZQUEZ, 2001) estos juegos y danzas pueden facilitar la inclusión del alumnado y el respeto hacia la diversidad. Mediante estas actividades, los niños y niñas inmigrantes pueden sentir valorados elementos de sus culturas de origen y pueden gozar de un protagonismo (si son ellos y ellas los que los explican a la clase) que quizás no gocen habitualmente. Paralelamente, el resto del alumnado puede perder el miedo a lo "desconocido y ex- 
traño" así como valorar la riqueza de otras culturas y romper estereotipos o prejuicios. Pero, como indican los mismos trabajos, es necesario que estas actividades formen parte de un proyecto educativo amplio y global del centro, lejos de ser un proyecto anecdótico y más bien folklórico con fecha de caducidad.

A continuación, se describen algunas experiencias y proyectos globales que persiguen la educación intercultural desde la Educación Física mediante un enfoque integral y permanente.

\section{Los juegos y bailes del mundo como punto de partida: algunas ex- periencias prácticas}

Una de las experiencias pioneras en Cataluña y que continúa desarrollándose hoy en día es la del "Rodajoc" (Ruedajuego). Desde 1997, en el barrio más multicultural de Barcelona, el barrio de Ciutat Vella, un grupo de profesores de Educación Física de educación primaria, promueve esta iniciativa (GARCÍA; PEDRET, 1999; PEDRET, 2004).

Ante el importante incremento de alumnado procedente de múltiples países en sus centros educativos (en alguno hay más de 80 nacionalidades representadas), el profesorado de Educación Física del barrio se propuso crear unas jornadas con el claro objetivo de fomentar la convivencia, el respeto y la tolerancia de una manera lúdica.

Durante el Rodajoc, en un parque del barrio, niños y niñas de 8 a 10 años forman grupos mezclándose por edades y centros (en el proyecto participan 13 escuelas). Todos los grupos van realizando un circuito de diversas estaciones en cada una de las cuales se realizan diferentes juegos del mundo aportados en muchos casos por el mismo alumnado: juegos de todos los continentes se juegan al mismo tiempo por parte de niños y niñas procedentes de todos los rincones del mundo.

Fruto del éxito del "Rodajoc" y a la implicación del profesorado, en el curso 2003-2004 nació una nueva experiencia, el "Mar de Jocs" (Mar de juegos), con la misma filosofía, pero con actividades y juegos en la playa destinados al alumnado de 6 a 8 años.

Esta experiencia es un ejemplo de colaboración permanente entre profesorado de diversos centros educativos y de regularidad. La constancia y tenacidad de las maestras y maestros que participan han permitido convertir estas jornadas en un auténtico encuentro de 
intercambio y comunicación entre los centros educativos y la comunidad del entorno, así como un ejemplo de convivencia.

Además de los juegos, otra actividad habitual que se utiliza en Educación Física son las danzas. Un buen ejemplo de ello es la experiencia que describe Boluda (2010) y que se realizó de forma interdisciplinar en un centro de educación primaria, entre las áreas de Educación Física y Educación Artística, bajo el nombre de "Les danses d'arreu del món" (Las danzas de alrededor del mundo).

El desarrollo de dicha experiencia pretendía implicar al alumnado en un proyecto conjunto entre ambas áreas, mediante la aportación de diferentes danzas culturales. Por ello, y de manera paralela, en el área de Educación Artística se trabajaron las diferentes músicas y danzas junto con su imprescindible contextualización, y en la Educación Física se crearon las diferentes coreografías en base a las músicas seleccionadas.

También de forma interdisciplinar, entre las áreas de Educación Física y Lengua Catalana, Ortí (2005) describe como, en un centro de secundaria, un grupo de jóvenes inmigrantes sin conocimiento del idioma elaboraron diferentes fichas de juegos -siendo necesaria una buena expresión lingüística-, para posteriormente llevarlos a la práctica. Mediante esta experiencia, se constató que la práctica de los juegos del mundo había desarrollado en los jóvenes, valores y actitudes de respeto y tolerancia, al mismo tiempo que les había facilitado el conocimiento del idioma y reforzado su autoestima.

Finalmente, otra experiencia relevante por la implicación que se consiguió de las chicas, fue la realizada en un centro de educación secundaria en el que el profesor observaba una baja participación femenina en las diferentes actividades físicas y diferentes problemas de integración para ellas. Ante este panorama, el docente elaboró una unidad didáctica llamada "El salto a la comba: yo, tú y todos y todas" con la intención de paliar las dificultades anteriormente comentadas y promover la coeducación, la convivencia y la interculturalidad (LLEIXA; SOLER, 2004). Mediante la introducción de la comba en sus clases, este profesor observó, dentro de un ambiente lúdico y participativo, un aumento en la motivación y en la cooperación entre el alumnado, que propició el intercambio cultural a través de la formación, de manera libre, de diferentes grupos mixtos y culturalmente diversos. 


\section{La administración local: la gestión de proximidad para la inclu- sión y la convivencia en y a través del deporte}

La nueva y cada vez más diversa realidad ciudadana ha llevado a todas las instituciones, desde el gobierno español a cada uno de los municipios, pasando por gobiernos autonómicos y provinciales, a pre(ocuparse) por la inclusión de la población inmigrante y el fomento de la convivencia.

Políticas deportivas y población inmigrante

Recientemente, el Consejo Superior de Deportes, responsable de las políticas deportivas a nivel del Estado español, ha incluido dentro del Plan Integral para la Actividad Física y el Deporte una línea de trabajo específica sobre "Inclusión social: Inmigración" (CSD, 2009)2. A nivel catalán, la Generalitat de Catalunya también ha promocionado el desarrollo de proyectos de inclusión y a nivel provincial, la Diputación de Barcelona, encargada de dar apoyo al desarrollo de los municipios, también ha incidido en ello. Así, para el trabajo a nivel local, la Diputación ha promovido varios cursos de formación de técnicos y técnicas en deporte y diversidad y ha creado la "Exposición itinerante de Juegos del Mundo" que, acompañada de una guía didáctica para facilitar la tarea educativa, se propone crear un punto de encuentro entre la ciudadanía y fomentar el respeto a la diversidad (Se puede ver en: http://www.diba.es/).

Precisamente es la administración local, la más cercana a la población, la que juega un papel más importante en el trabajo de la convivencia e inclusión, en y a través de la práctica deportiva. Para ello, resulta imprescindible la transversalidad vertical -entre distintos niveles de la administración-, así como la transversalidad horizontal -entre las distintas áreas del municipio, como la de deportes, inmigración, mujer, juventud, etc. -.

El equipo de investigación del Centre d'Estudis Olímpics de la Universitat Autònoma de Barcelona ha realizado, en los últimos años, varios estudios sobre el multiculturalismo en las políticas deportivas locales. De ellos, se desprende que la postura asumida por la adminis-

2-Fruto de este programa, se ha elaborado la Guía: Deporte, Actividad Física e Inclusión Social. Una guía para la intervención social a través de las actividades deportivas (MAZA, 2011).

Pensar a Prática, Goiânia, v. 15, n. 1, p. 1-271, jan./mar. 2012 
tración local es, básicamente, "facilitar el acceso de la población extranjera a la oferta deportiva existente como cualquier otro ciudadano" (KENNET; SAGARZAZU; CEREZUELA, 2007, p. 37). En el mismo trabajo, así como en el estudio a nivel europeo promovido por la Dirección General de Educación y Cultura de la Unión Europea (PMP y INSTITUTE OF SPORT AND LEISURE POLICY OF LOUGHBOROUGH 2004), se señala que las actuaciones dirigidas a fomentar la cohesión social y la integración de personas extranjeras denotan un cierto carácter asimilacionista en el que los y las inmigrantes se adaptan a la oferta existente, siendo necesario en algunas ocasiones, cambiar sus hábitos. Esta tendencia puede dar lugar a que "la población extranjera se niegue, desconozca o no consiga adaptarse a la oferta deportiva local" (KENNET; SAGARZAZU; CEREZUELA, 2007, p. 37). Por otra parte, la misma investigación señala que las mujeres inmigrantes son el colectivo menos atendido en las políticas deportivas públicas (KENNET; SAGARZAZU; CEREZUELA，2007; SAGARZAZU, 2007).

En este contexto, se considera necesario conocer las prácticas deportivas que lleva a cabo la nueva población, cuáles son sus hábitos deportivos, en qué se parecen y en qué no se parecen a la población general, en qué espacios y de que modo practican deporte y como incide la perspectiva de género.

Diferentes y no tan diferentes formas de ver y vivir el deporte: hábitos deportivos, uso del espacio público y la perspectiva de género entre la población inmigrante

La gran diversidad de procedencias de la nueva ciudadanía de Cataluña hace que el repertorio de deportes y actividades físicas que han llegado al territorio con la población inmigrante sea muy amplio y variado. Chris Kennet y Kim Manresa (2008) nos dan buen ejemplo de ello en su trabajo de fotoperiodismo e investigación social en el que recogen algunas de las actividades que practican los y las inmigrantes en Cataluña (se pueden ver en la exposición fotográfica en línea: Deporte multicultural). En ella se encuentran algunas de las nuevas prácticas desconocidas en Cataluña, como el kabaddi -llegado de la Indiao los deportes gaélicos, así como deportes que hasta hace poco eran muy minoritarios pero han experimentado un importante incremento de participantes. Son ejemplo de ello la expansión del béisbol -especi- 
almente practicado por jóvenes sudamericanos- o el cricket -muy extendido entre la población pakistaní- y que está en proceso de institucionalización.

Todas estas prácticas se añaden a las actividades tradicionales catalanas y a los deportes más extendidos. En esta línea, como apuntan Jaume Bantulà y Ricardo Sánchez (2008), la llegada de población inmigrante ha supuesto un incremento de licencias federativas de un 10 a un $30 \%$ en deportes como la lucha, el voleibol, el ajedrez o el béis$\mathrm{bol}^{3}$. No obstante, es en la práctica informal a nivel local donde se observa una mayor participación.

En el caso de la ciudad de Barcelona, según el trabajo de Bantulà y Sánchez (2008), la actividad deportiva de la población extranjera se caracteriza por realizarse en el espacio público, de modo informal, como diversión, con familiares o grupos de amistades, y sin inscribirse a un club o asociación. Se constata, también, que la práctica deportiva se convierte en una forma de reforzar la identidad cultural y nacional, y que hay claras diferencias por género y edad en el tipo de actividades que se realizan y el nivel de participación.

Observando estos datos, los autores del estudio destacan que, a pesar de que a menudo se trata como una realidad diferenciada, lo cierto es que las y los inmigrantes muestran las mismas tendencias en la práctica deportiva que el resto de la población de la ciudad según la última encuesta de hábitos deportivos de Barcelona (AYUNTAMIENTO DE BARCELONA, 2006): un $77 \%$ de practicantes no pertenece a ningún club o asociación y el $62 \%$ utiliza de forma regular espacios como la calle, los parques, la playa, etc. Las principales diferencias se observan, entonces, en que la población extranjera tiende a practicar deporte en grupo y por divertirse mientras que la población en general tiende a realizar deporte de forma individual y por mantenerse en forma, más dentro de la lógica del proceso de individualización de la sociedades contemporáneas (BANTULÀ; SÁNCHEZ, 2008).

Es en el espacio público, entonces, donde la mayor parte de la población -inmigrante o no-, practica deporte. Xavier Camino (2007), en su estudio sobre el uso deportivo del espacio público de Barcelona, constató que:

3-En su trabajo los autores no mencionan el fútbol, si bien la participación de población inmigrante en este deporte es también muy relevante.

Pensar a Prática, Goiânia, v. 15, n. 1, p. 1-271, jan./mar. 2012 
Las redes de inmigrantes extranjeros, como los nacionales en sus inicios, suelen ir cambiando de espacios por toda la ciudad esperando no ser expulsados por las autoridades que responden a quejas vecinales. Por eso buscan descampados alejados del vecindario donde poder desarrollar sus actividades sin ocasionar preocupaciones a los vecinos, aunque no siempre es posible. El encuentro se realiza sólo los fines de semana y los festivos durante todo el día. Practican deportes propios de su país de origen tales como el ecuavolei, el críquet y el softball. Estos encuentros permiten el mantenimiento de una cohesión social lejos del país de origen y son muy importantes como elemento de apoyo material y emocional. (CAMINO, 2007, p. 546).

Además, tal y como recogen Camino, Maza y Puig (2008), el espacio público se convierte en un contexto privilegiado para la creación de redes sociales mediante la realización de un amplio espectro de actividades deportivas. La conexión y la interacción con otras personas mediante el deporte, pues, permite incrementar el capital social, que es uno de los elementos clave para alejarse del riesgo de exclusión social (MAZA, 2002; 2004). En este sentido, desde el Plan Integral para la Actividad Física y el Deporte (CSD, 2009), se considera que el deporte en el espacio público es una de las principales líneas estratégicas a promover. Como apuntan Marta Carranza y Carles Vallès (2008) para el caso de Barcelona, el hecho de favorecer la práctica deportiva en los espacios públicos permite acercar el deporte a toda la población y especialmente a los colectivos con más dificultades económicas y de acceso a las instalaciones deportivas convencionales.

Sin embargo, en el espacio público, así como en las instalaciones deportivas convencionales, se constata una gran ausencia, la de las mujeres inmigrantes practicando deporte (VILANOVA; SOLER, 2008; SAGARZARZU, 2007).

El deporte tradicional es utilizado frecuentemente como una actividad que puede facilitar la integración de los hombres recién llegados procedentes de otras culturas y países, pero no siempre es una herramienta adecuada para facilitar la integración de las mujeres. Por otra parte, en algunas culturas, los equipamientos deportivos acostumbran a ser espacios vedados para las mujeres, y resulta difícil que en su llegada aquí empiecen a utilizarlos sin medidas que lo faciliten.

El colectivo de mujeres inmigrantes es muy diverso, y la situación 
de cada mujer puede variar mucho según su trayectoria migratoria. Sin embargo, la mayor parte de las mujeres inmigrantes acostumbra a encontrarse con dificultades para romper con el aislamiento en que se encuentra al llegar, ya sea debido a las barreras lingüísticas como a las características de su jornada laboral (habitualmente en el servicio doméstico) y las cargas familiares. A pesar de ello, no siempre interiorizan una imagen victimizadas de ellas mismas sino que desarrollan modelos positivos. No obstante, a menudo sus condiciones de vida están marcadas por la desvalorización social y el acceso desigual a los recursos de la sociedad. Este hecho, junto a la situación de desarraigo y desamparo social y económico en la que se encuentran, puede conducir a estas mujeres a situaciones de frustración, sentimiento de inferioridad, baja autoestima, etc.

Ante esta situación, la práctica de actividades que respondan a sus intereses puede ser una herramienta más para el desarrollo de su autoestima y la mejora de la calidad de vida. Para ello, tal y como se ha constatado mediante diversas experiencias (SANTOS et al., 2004; PFISTER, 2004; SOLER, 2007; SOLER, GAZTELU; SERRA, 2010), se trata de plantear actividades que tengan en cuenta las necesidades de las mujeres y los aspectos de salud, relación social y ocio sean abordados desde sus expectativas y las circunstancias en que se encuentran.

En definitiva, conocer las formas de ver y vivir el deporte de la nueva ciudadanía, recoger sus intereses y necesidades teniendo en cuenta su gran diversidad, darles cabida en el sistema deportivo local sumando propuestas y entidades, y gestionar el espacio público para el uso deportivo son aspectos que sin duda deben abordar las administraciones locales.

\section{A modo de conclusión}

La escuela y la administración local son las instituciones más cercanas a la población y las que, a primera línea, deben dar respuesta a una nueva realidad ciudadana cada vez más diversa y cambiante. Desde ambas instituciones, la Educación Física y el deporte pueden ser herramientas muy útiles para favorecer la inclusión, la convivencia y la interculturalidad, siempre y cuando se utilicen de forma adecuada.

En este artículo, se han presentado varios estudios y experiencias que muestran como se está realizando este trabajo en Cataluña. En to- 
dos ellos se constata la importancia de tres principios básicos a tener en cuenta en la planificación: la participación, la transversalidad y la continuidad.

Para favorecer la inclusión del alumnado en la escuela, así como del conjunto de la población inmigrante en el municipio a través del deporte, hombres y mujeres, es imprescindible hacerles partícipes de las propuestas o proyectos y recoger sus aportaciones y formas de ver y vivir la actividad física y el deporte. Mediante este proceso participativo es posible incorporar la amplia gama de culturas deportivas existente en la Educación Física y el deporte y dar un nuevo enfoque a la oferta y a los espacios deportivos.

Por otra parte, cuando las propuestas se realizan de forma transversal, desde diferentes instituciones y desde diferentes áreas o disciplinas, ya sea a nivel escolar o municipal, también hay muchas más posibilidades de éxito. Se trata pues, de abordar la problemática de forma integral: aunar los esfuerzos de las diversas instituciones de la zona (ayuntamiento, centros educativo, clubs y entidades deportivas, asociaciones, etc.), incorporar los conocimientos y propuestas de las diferentes áreas educativas o del municipio, y tener en cuenta tanto la perspectiva de quién toma las decisiones en los despachos como de quién las lleva a cabo en el patio o la calle.

Y finalmente, se constata la importancia de buscar mecanismos que den continuidad a los proyectos, ya que las actuaciones puntuales pueden facilitar la sensibilización y el conocimiento mutuo, pero corren el riesgo de quedar en meras anécdotas si no se realizan con constancia y regularidad. Por otra parte, el trabajo continuo permite ir reajustando los proyectos de un año a otro, mejorando lo que se considere necesario e incorporando nuevas propuestas a partir de la reflexión sobre la propia actuación y la participación de todas las personas y agentes implicadas.

Desde la proximidad que tienen la escuela y la administración local su tarea es esencial para abordar con éxito la inclusión de las personas que llegan a Cataluña $u$ a cualquier otro territorio. Mediante la Educación Física y el deporte, ambas instituciones pueden y deben crear espacios de encuentro e interacción que faciliten este objetivo. 
Physical education and sports as tools for inclusion of the immigrant population in Catalonia: the role of scholl and local administration

\begin{abstract}
There are two institutions which are the most close to citizens with a key role to promote the inclusion of the immigrant population: the school and the local administration. This article sets out the main actions that are carried out from both fields to take advantage of the socializing potential of sport and physical activity in Catalonia. We describe the political approach which guide both institutions and some experiences and data. From this analysis, we can remark the importance of designing strategies and proposals for intervention taking into account the basic principles of participation, mainstreaming and continuity.

Keywords: Physical Education. Sports. Immigration. Inclusion.

Educação física e esporte como ferramenta de inclusão da população imigrante na Catalunha: o papel das escolas e do governo local

\section{Resumo}

Há duas instituições que desempenham um papel fundamental na promoção da inclusão da população de imigrantes da vizinhança da cidadania: a escola é a administração local. Este artigo descreve as principais ações realizadas partindo de ambas área aproveitando o potencial de socialização do esporte e da atividade física na Catalunha. Com base na abordagem política que guia as atividades educativas e de nível municipal, descreve algumas experiências nas duas áreas. Como resultado desta análise, podemos notar a importância das estratégias e propostas de intervenção tendo em conta os princípios básicos da participação, transversalidade e continuidade.
\end{abstract}

Palavras chave: Educação Física. Esportes. Imigração. Inclusão.

\title{
Referências
}

AGUDO, D. et al. Juegos de todas las culturas. Juegos, danzas, música... desde una perspectiva intercultural. Barcelona: Inde, 2002

AYUNTAMIENTO DE BARCELONA. Hàbits esportius a Barcelona. Barcelona: Institut Barcelona Esports, 2006.

BANTULÀ, J.; MORA, J.M. Juegos multiculturales. 225 juegos tradicionales para un mundo global. Barcelona: Paidotribo, 2002.

BANTULÀ, J.; SÁNCHEZ, R. Inmigración y culturas deportivas: estudio para determinar los criterios de intervención y promoción deportiva municipal en Barcelona. En: CANTARERO, L.; MEDINA, X.; 
SÁNCHEZ, R. Actualidad en el deporte: Investigación y aplicación -X Congreso de Antropología. Donosti: Ankulegui, 2008.

BOLUDA, G. Les danses d'arreu del món: Una proposta interdisciplinària per gaudir de la diversitat cultural al nostre centre. Guix, Barcelona, n. 364, 2010, p. 23-27.

CAMINO, X.; MAZA, G.; PUIG, N. Redes sociales y deporte en los espacios públicos de Barcelona. Apuntes. Educación física y deportes. Barcelona, n. 91, 2008, p. 12-28.

CAMINO, X. Los espacios públicos urbanos y el deporte como generadores de redes sociales. El caso de la ciudad de Barcelona. En: MIRAGAYA, A. et al. Universidad y estudios olímpicos: Seminarios España-Brasil 2006. Bellaterra: Centre d'Estudis Olímpics, 2007, p. 538-550. Disponible en: http://doc.rero.ch. Consultado: Septiembre 2011.

CAPLLONCH, M.; GODALL, T.; LLEIXÀ, T. El professorat d'educació física a l'escola multicultural. Percepcions del context i necessitats de formació. Temps d'educacio. Barcelona, n. 33, 2007, p. 61-74.

CATALUÑA. Ley 12/2009, de 10 de julio. Llei d'educació de Catalunya. Diari Oficial de la Generalitat de Catalunya, Barcelona, No. 5422, p. 56589- 56682, 10 de juliol de 2009.

CARRANZA, M.; VALLÈS, C. El deporte en la ciudad: una nueva mirada de verlo. Apuntes. Educación física y deportes. Barcelona, $\mathrm{n}$ 91, 2008, p. 9-10.

COMISIÓN EUROPEA. Libro blanco sobre el deporte. Bruselas, 2007. Disponible en: http://ec.europa.eu/sport/. Consultado: Septiembre 2011.

CONSEJO SUPERIOR DE DEPORTES. Plan Integral para la Actividad Física y el Deporte. Madrid, 2009. Disponible en $<$ http://www.csd.gob.es/csd $>$. Consultado: Setiembre, 2011.

CUEVAS, R. La educación intercultural a través del movimiento. Revista Aula De Innovación Educativa [Versión Electrónica]. Barcelona, n.181, 2009. 
DEPARTAMENT D'EDUCACIÓ. Currículum educació primària. Barcelona: Generalitat de Catalunya. 2009a. Disponible en: http://phobos.xtec.cat/. Consultado: Octubre 2010.

DEPARTAMENT D'EDUCACIÓ. Plan para la lengua y la cohesión social, educación y convivencia intercultural. Barcelona: Generalitat de Catalunya, 2009b. Disponible en: http://www.xtec.cat/lic/. Consultado: Febrero 2010.

DEPARTAMENT D'EDUCACIÓ. Avenç dades curs 2010-2011. Barcelona: Generalitat de Catalunya, 2011. Disponible en: http://www20.gencat.cat/docs/. Consultado: Octubre 2011.

EXPÓSITO, J. et al. La educación física, vehículo para erradicar prejuicios culturales. Revista Aula De Innovación Educativa [Versión Electrónica]. Barcelona, n.82, 1999.

GARCÍA, R.; SALES, A. Educación intercultural y formación de actitudes. Programa pedagógico para desarrollar actitudes interculturales. Revista Española De Pedagogía. Madrid, n.207, 1997, p. 317-336.

GARCÍA, C.; PEDRET, R. "Rodajoc": La experiencia de juego sin fronteras. Revista Aula De Innovación Educativa [Versión Electrónica]. Barcelona, n. 82, 1999.

GARCÍA, C.; MARTÍNEZ, A. El juego de las cuatro esquinitas del mundo: Libro de juegos para favorecer las relaciones interculturales. Madrid: Catarata, 2004.

GRANDA, J.; DOMÍNGUEZ, R.; EL QUARIACHI, S. El juego popular y tradicional como mediador intercultural: Una propuesta educativa para favorecer las relaciones interculturales a través del juego, el respeto y la convivencia interétnica. Barcelona: Cims, 1999.

HEINEMANN, K. Deporte para los inmigrantes: ¿instrumento de integración? Apuntes. Educación física y deportes. Barcelona, n. 68, 2002, p. 24-35.

IDESCAT. Evolución de la población total y extranjera, 2000-2010. Barcelona, 2011. Disponible en: http://www.idescat.cat/. Consultado: Septiembre 2011. 
KENNETT, C.; MANRESA, K. Deporte multicultural. Barcelona: Ajuntament de Barcelona \& Centre d'Estudis Olímpics, 2008.

KENNETT, C.; SAGARZAZU, I.; CEREZUELA, B. EI multiculturalisme en les polítiques esportives: anàlisi de l'adequació de les polítiques locals a Catalunya a les actuacions promogudes per la Unió Europea. Centre d'Estudis Olímpics, 2007. Disponible en: http://olympicstudies.uab.es. Consultado: Septiembre, 2011.

LLEIXÀ, T.; SOLER, S. Actividad física y deporte en sociedades multiculturales. ¿Integración o segregación? Barcelona: ICE-Horsori, 2004.

MAZA, G. El deporte y su papel en los mecanismos de reproducción social de la población inmigrante extranjera. Apuntes. Educación física y deportes. Barcelona, n. 68, 2002, p. 58-66.

. El capital social del deporte. En: LLEIXÀ, T; SOLER, S. Actividad física y deporte en sociedades multiculturales. ¿Integración o segregación? Barcelona: ICE-Horsori, 2004, p. 43-56.

MORA, J.M.; DÍEZ, R.; LLAMAS, J. Un mundo en juego. Barcelona: INDE, 2003.

ORTÍ, J. La educación física y el juego en la integración del alumnado inmigrante. Revista Aula de Innovación Educativa [Versión Electrónica]. Barcelona, n.140, 2005.

PEDRET, R. Las actitudes, valores y normas en el espíritu del rodajoc. Revista Aula De Innovación Educativa [Versión Electrónica]. Barcelona, n.136, 2004.

PFISTER, G. Deporte, género y etnicidad: el caso de las mujeres inmigrantes. Apuntes. Educación física y deportes. Barcelona, n. 78, 2004, p. 117-124.

PMP \& INSTITUTE OF SPORT AND LEISURE POLICY OF LOUGHBOROUGH. Sport and multiculturalism: final report: Studies on education and sport. European Commission - DG Education $\&$ Culture, 2004. Disponible en: <http://www.isca-web.org/> Consultado: Septiembre, 2008. 
PRAT, M.; SOLER, S. Las posibilidades de la educación física y el deporte para la mejora de la convivencia. Revista electrónica interuniversitaria de formación del profesorado. v.5, n.2, 2002. Disponible en: http://www.aufop.com/. Consultado: Septiembre 2011.

. Actitudes, valores y normas en la educación física y el deporte. Reflexiones y propuestas didácticas. Barcelona: INDE, 2003.

SAGARZAZU, I. Mujer inmigrante y deporte. En: MIRAGAYA, A. et al. Universidad y estudios olímpicos: Seminarios España-Brasil 2006. Bellaterra: Centre d'Estudis Olímpics, 2007, p. 52-62. Disponible en: http://doc.rero.ch. Consultado: Septiembre 2011.

SÁNCHEZ, J.; MARTÍN, J. Bailes del mundo: Una propuesta de bailes populares para educación primaria. Barcelona: Paidotribo, 2002.

SANTOS, A. et al. Mujeres en forma contra la exclusión. Valencia: Delegación general de la mujer, 2004.

SOLER, S. El programa "Bellugant-nos": actividad física para mujeres inmigrantes y autóctonas del barrio Collblanc - Torrassa (Hospitalet). En: MIRAGAYA, A. et al. Universidad y estudios olímpicos: Seminarios España-Brasil 2006. Bellaterra: Centre d'Estudis Olímpics. 2007, p. 42-52. Disponible en: http://doc.rero.ch. Consultado: Septiembre 2011.

SOLER, S. Los procesos de reproducción, resistencia y cambio de las relaciones tradicionales de género en la Educación Física: el caso del fútbol. Cultura \& Educación. Madrid, v.21, n.1, 2009, p. 23-43.

SOLER, S.; GAZTELU, M.; SERRA, P. Disseny, implementació i avaluació d'un programa d'activitat física per a la inclusió femenina a L'Hospitalet de Llobregat. 2010. Memoria del projecto ARAFI 2009. Disponible en: http://inefcgiseafe.wordpress.com/. Consultado: Septiembre 2011.

VELÁZQUEZ, C. Los juegos y danzas del mundo como recurso para una educación física intercultural. Una propuesta en educación primaria. Revista Tándem [Versión Electrónica]. Barcelona, n.5, 2001.

. 365 juegos de todo el mundo: Juegos para construir un mundo mejor. Barcelona: Océano Ambar, 2006. 
VILANOVA, A.; SOLER, S. Las mujeres, el deporte y los espacios públicos: ausencias y protagonismos. Apuntes. Educación física y deportes. Barcelona, n.91, 2008, p. 29-34.

Recebido em: 23/12/2011

Revisado em: 15/01/2012

Aprovado em: 24/02/2012

\section{Endereço para correspondência}

ssoler@gencat.cat

Susanna Soler Prat

Instituto Nacional de Educación Física de Cataluña

Av. de l'Estadi 12-22

Anella Olímpica

08038 Barcelona - España 